\author{
SERIES 'CHEST PHYSICAL EXAMINATION' \\ Edited by J.C. Yernault
}

\title{
Acoustic properties of the normal chest
}

\author{
F. Dalmay, M.T. Antonini, P. Marquet, R. Menier
}

Acoustic properties of the normal chest. F. Dalmay, M.T. Antonini, P. Marquet, R. Menier. (C) ERS Journals Ltd 1995.

ABSTRACT: Laënnec invented the stethoscope in 1816 and published a treatise on auscultation in 1819. We then had to wait until the 1950s to observe development of modern devices and methods of recording and signal-processing, which allowed objective studies of lung sounds in time and frequency fields.

Tracheobronchial sounds generated by ventilation originate in the upper airways, the frequency content of these sounds has led to extensive research. Consolidated lungs act as more efficient sound conductors to the chest wall (bronchial breathing murmur). Tracheobronchial sounds contain higher frequency components compared to vesicular lung sounds.

The origin of vesicular lung sounds has been becoming progressively clear for about 10 yrs. It is at least partly produced locally, deep, and probably intralobular. Clearly, further studies need to be performed in order to elucidate the true mechanisms involved in generating vesicular lung sounds, the redistribution of intrapulmonary gas or vibrations caused by the stretching of lung tissue.

The devices developed are already useful for monitoring the state of patients in intensive care. Sooner or later, real time analysis and automated diagnosis will become available.

Eur Respir J., 1995, 8, 1761-1769.
Laboratoire de Physiologie, Faculté de Médecine, Limoges, France.

Correspondence: R. Menier CHRU Dupuytren

Physiologie et Exploration Fonctionnelle 2, rue Martin Luther-King 87042 Limoges Cedex France

Keywords: Normal lung sounds sensors

signal-processing

tape-recording

tracheobronchial sounds

vesicular lung sounds

Received: June 101994

Accepted after revision March 311995
Laënnec invented the stethoscope in 1816 and published his treatise on auscultation in 1819, describing acoustic events generated by ventilation of the lungs and systematically correlating them with anatomical and pathological findings after autopsy. "Auscultation of breathing sounds with a cylinder (stethoscope), produces easily interpreted auditory signals capable of indicating the presence and extent of most disorders of organs in the thoracic cage...". Auscultation of healthy individuals enables "ventilatory murmur" (i.e. vesicular breath sounds) to be heard, and Laënnec goes on to describe a range of abnormal sounds associated with different pathological states. This form of semiology, however, is not without a number of drawbacks; it lacks sufficient sensitivity, offers no quantitative measurement of the sounds detected, is essentially a subjective clinical test, and is hindered by an imperfect system of nomenclature. However, subsequent development of X-ray radiography was so far reaching that Forgacs [1] was able to comment in 1969 that "the success of radiography in revealing structural changes in the lungs had a profound effect on the clinical approach to diseases of the chest. It turned the interest of physicians towards morphology at the expense of function and led to the decline in the status of auscultation of the chest from its key position to a perfunctory ritual". Twenty five years later, the question remains of why anyone should wish to further investigate the acoustic properties of the lung hoping to discover new diagnostic applications for auscultation.

In fact, pulmonary auscultation is widely available at the bedside; it is cheap, quick, easy to carry out and to repeat, noninvasive and totally innocuous. This is of particular advantage when dealing with the newborn and young infants as well as the old-aged, mental patients and unconscious subjects, for all of whom conventional respiratory function tests can prove difficult or impossible.

Since the 1950s, techniques and equipment have been developed that improve the detection and analysis of respiratory sounds. This has opened the door to objective studies of such signals [2, 3], facilitating precise topographical localization of lung sounds and clarification of their aetiology. It therefore seems likely that, sooner or later, real time analysis and automated diagnosis should become available and that the first to benefit from this type of monitoring will probably be patients on intensive care wards.

\section{Historical summary}

Direct auscultation was already known to Hippocrates, who advised application of the ear to the patient's chest in order to hear sounds transmitted through the chest 
wall. However, by the time Laënnec first started practising medicine, at the beginning of the 19th century, the technique had practically fallen out of favour and, indeed, the very idea seems to have revolted him. Inspiration for his invention came one day, when he noticed two children sending messages to one another along a wooden stick, alternately scraping one end of the stick and then pressing it to an ear to hear the reply [4]. Laënnec proceeded to experiment with various wooden cylinders and rods, finding to his satisfaction that a solid rod placed between his ear and the patient's chest significantly improved sound transmission, and that a rod pierced with a narrow bore was an even more effective sound conductor.

Clinical examination of pulmonary function was divided at that time into four separate stages: questioning (i.e. taking the patient's history), inspection, palpation and percussion. Direct percussion had only been introduced shortly before, palpation serving to evaluate transmission of vocalized vibrations from the trachea through to the chest wall.

Laënnec initially sought to use his cylinder stethoscope to facilitate detection of whispered sounds transmitted to the chest wall. He found that, in healthy subjects, whispering produces a sound of low intensity accompanied by a slight distortion of the voice, being most audible over the axillae and scapulovertebral regions, as well as opposite sternoclavicular articulations.

He also noted that pleural effusion and pulmonary consolidation prevented the passage of sound waves, whereas transmission increased when he placed his "cylinder" over tuberculosis cavitations or areas of underlying bronchiectasis. Major cavitations resulted in identical vocalized sounds (pectoriloquy) heard through the chest wall and through the larynx. High-pitched sounds travelled best. Laënnec associated one particular type of sound, egophony (a noise resembling the bleating of a goat) with limited liquid pleural effusions.

He next turned his attention to the study of "spontaneous" open-mouth breathing, to further develop his technique for "auscultation of breathing sounds with the aid of the cylinder". Healthy subjects generated vesicular lung sounds, the "murmur of respiration", whilst patients with pathological conditions produced adventitious lung sounds" ...sufficiently distinctive to permit identification of most organic disorders of the chest".

It was not long before several models of stethoscopes were elaborated by other medical workers. Comyns in the USA invented the binaural stethoscope (see [5]), in which sound was channelled through tubing to both ears from a single membrane mounted in a flat-cup chest piece. Alison (see [6]) in London, created a modified version of the stethoscope in 1861, that he called the symballophone. It consisted of two terminal cups or bells that could simultaneously capture sounds from two different points on the chest and, consequently, detect delays in the propagation of vibrations through the chest tissues or allow comparison of relative noise levels.

The stethoscopes employed today for auscultation of the heart or lungs, are equipped with membranes that give a good frequency response, connected to rubber tubing with poor transmission properties which conduct sound directly to the ears [7]. The resulting overall sensitivity is consequently low with an uneven frequency response, falling off by 3-6 dB per octave above 100 $\mathrm{Hz}$. "A good quality stethoscope should have a flat response curve with less than $\pm 3 \mathrm{~dB}$ variation between 50 and $1,200 \mathrm{~Hz}$ and should provide high sensitivity over this range" [7].

\section{Modern methods of recording and signal-processing}

"Lung sounds result from the vibrations within the lung and its airways that are transmitted to the chest wall. Vibration amplitude may be less than $10 \mu \mathrm{m}$ and is affected by the method by which it is determined or transduced" [8].

Hannon and Lyman (see [8]) were probably the first to use a mechanico-electrical transducer to detect lung sounds. Their method involved recording with a microphone linked through filters to a string oscilloscope and, thence, to a graphic recording device.

In 1955, McKusicK et al. [9] employed condenser-type microphones and recorded the resulting electrical signals on a magnetic disc, which could be read several times successively, each time scanning at a different frequency with a variable filter. The corresponding signal strength of each frequency band was then translated into a proportionate level of light intensity, and the data thus recorded on light-sensitive paper.

Most subsequent studies followed a similar general pattern of steps: detection, preliminary processing, recording, and final processing of recorded signals. Equipment and technique, however, varied widely from one study to another and this diversity prevents any meaningful comparison of results.

"Electronic recording and computer analysis of respiratory sounds from the chest and trachea of normal subjects and patients with respiratory disease, has, over the past decade or so, become a major preoccupation of many clinical and engineering teams" [10].

The principal objective of such research is to increase understanding of the fundamental mechanisms that cause breathing sounds [11-17]. The second objective is to evolve a system of classification that will enable automated processing of normal and pathological lung sounds. In this way, early diagnosis of pathological signs would gain in accuracy and reliability compared to the wholly subjective procedures of conventional auscultation using a stethoscope [18-21].

Equipment for the analysis of heart sounds is already available on the market and regularly used in clinical practice. This is not surprising, since heart sounds are loud, they originate from a discrete region close to the chest wall, are emitted within a range of low frequencies [22], and can be recorded whilst the subject holds his or her breath, thus excluding interference from extraneous lung sounds.

Similar systems for analysing lung sounds are still in their early stages of development. The situation is considerably more complicated in pneumology; lung sounds 
are spread over a much wider frequency band and depend upon several factors, such as airflow rate, inspiration or expiration phases, site of recording, degree of voluntary control that the subject is able to exert over breathing, and interference from heart sounds [22].

Another major obstacle to progress is the diversity of procedures and signal-processing techniques adopted by researchers. Differences in instrumentation, treatment and presentation of acquired data render comparison of individual reports extremely difficult. There is an obvious need for agreement over standardization of recording and information-processing techniques [10].

A typical system for recording lung sounds consists of a sensor housed in a suitable support that picks up sound vibrations at the surface of the chest wall or over the trachea. Analogue signals from the microphone and from an airflow transducer can then be simultaneously recorded on magnetic tape, or fed directly to a computer for storage in digital form. Alternatively, the tape can be read back and signals, after sampling and digitizing, can be transferred to the computer for analysis.

\section{Sensors}

The first types of sensors used to record lung sounds were merely amplified stethoscopes, but high levels of background noise and frequency band distortion soon led to their rejection. Experiments with carbon microphones, cord galvanometers and various optical devices also proved unsatisfactory [23].

The microphone represents a critical part of the electromechano-acoustic bridge between recording instrumentation and subject and, consequently, its type and quality are of extreme importance. Theoretical properties of all types of sensor are well-documented [23], information being available on such factors as sensitivity, dynamic range, frequency response, harmonic and phase distortion, transient response, signal-to-noise ratio, susceptibility to ambient noise, reproducibility of signal response and sensitivity to static forces.

Most researchers in the 1970s chose to work with phonocardiographic microphones [24, 25] although it rapidly became clear that they were ill-adapted to the low sound levels and relatively broad spectrum of frequencies generated by breathing. By the mid-1970s, other types of microphone were being adopted; condenser and externally polarized microphones $[6,12,26-30]$, or electret microphones [23, 31-34]. A few research teams experimented with dynamic microphones [30], accelerometers, and various piezoelectric contact microphones [35-38], but the most suitable type was generally considered to be the condenser microphone [23].

Calibration of microphones can be carried out by means of a fixed value sound pressure source, or an arbitrary level reference sound, or by comparison with a standard reference microphone. However, most researchers choose not to calibrate their microphones, preferring instead to express results in terms of relative amplitude, whilst some teams calibrate the entire sound recording system [11, $23,28,40]$.
Recording generally takes place without the provision of a soundproof chamber and, as a result, the performance characteristics of the microphones used depend to a large degree upon effective mechanical coupling of the sensor to the subject's skin, aiming for maximum sensitivity to breathing sounds, yet with as little interference as possible from ambient background noise. Coupling devices have included: 1) the circular plastic bell-endpiece from a stethoscope $[27,41]$; 2) a cylindrical probe made of polyvinylchloride (PVC) [29]; 3) a plastic cone $[22,23]$; 4) a rubber diaphragm fitted over a metal alloy tube [32]; and 5) a small plastic tube [21, 34].

The design of acoustic coupling devices requires great care. Microphone shrouds and attachments are known to cause resonance peaks, and designs should be modified to ensure that no interference occurs inside the range of frequencies to be studied.

A number of different methods have been employed to fix microphones to the chest wall. They can be: handheld; fixed by an elastic or nonelastic strap; glued in place, sometimes by means of a double-sided adhesive ring [23, 28, 42-44]; or attached by suction pads. No entirely satisfactory system has yet been found, because of the constantly changing properties of soft intercostal tissues during different stages of the ventilatory cycle.

\section{Initial processing of raw data}

Before storing and analysing, signals from sensors pass through an initial processing step that includes preamplification and filtering, both of which increase the risk of deforming useful components of the original signal. Low output voltage from microphones (10-200 mV) must be amplified to a suitable input level for treatment by analogue-to-digital converters (A/D converters) or recording on a magnetic support. It is, therefore, necessary to use a good quality low-noise amplifier with a flat frequency response curve, adequate gain $(500-1,000)$ and input impedance values correctly matched to microphones. These technical considerations become particularly relevant whenever microphone lines and wiring are longer than a few dozen centimetres. Perhaps the best way to reduce signal loss and noise is to incorporate an impedance adapter within the housing of the microphone itself [23], though despite progress in miniaturization, this has the inconvenience of adding extra weight to the assembly.

Manufacturers of specialist equipment will generally have selected the most appropriate amplification stages to match the types of sensors provided [7, 11-13, 27, 29, 45].

The use of high-pass filters between microphone and recorder is another area of unresolved dilemma. The filters are designed to eliminate low-pitched frequencies, which because of their high energy are liable to saturate recorder input channels or, at the very least, cause a reduction in signal headroom, thus compressing response at higher frequencies. Although respiratory sounds are 
generally considered to contain a small proportion of lower frequencies, most of them are due to noise from the heart and muscles [22, 46]. However, to our knowledge, no study has either confirmed or disproved the conjectured contribution of breathing sounds to this lower end of the frequency band. Filter cut-off frequencies used in experimental work have ranged $50-200 \mathrm{~Hz}$, with attenuation values of $12-80 \mathrm{~dB}$ per octave, but so far no standard value has been proposed for either of these criteria. Indeed, the technical characteristics of filters often go unreported and little attention is given to factors such as linearity of phase with respect to frequency, even in work devoted to the study of phase in breathing sounds. Recent publications have described sophisticated approaches to discriminating between heart and pulmonary sounds, for example the adaptive filtering technique [47, 48].

Certain authors also add a low-pass filter to their analogue recording system, so that background noise can be further reduced and time-amplitude signal representation improved. If so wished, the task of selectively removing unwanted high and low frequencies can be handled simultaneously by fitting a single band-pass filter.

\section{Tape-recording}

Most reports since the 1960s have described systems which include multitrack tape machines, initially using amplitude modulated (AM) then frequency modulated (FM) carriers.

Limited memory storage capacity has meant that signals are usually treated in two distinct stages: processed signals are first recorded on tape; and the tape is then played back in short segments to allow conversion of signals into digital data. This dual-step process offers the advantage of keeping recording equipment light and portable, and also allows longer sessions of breathing sounds to be recorded. On the other hand, repeated playback of tapes inevitably leads to physical deterioration of the magnetic support and consequent loss of sound quality.

Improved cassette tape decks have given amplitude modulation recording a new lease of life over the last decade and have progressively been adopted as monitoring devices on intensive care wards [28, 33, 37, 41].

$\mathrm{AM}$ recorders have a frequency response ranging 50 $\mathrm{Hz}$ to $15 \mathrm{kHz}$, whereas FM recorders cover a much wider spectrum extending from virtually $0 \mathrm{~Hz}$ to upper limits which can vary between $5-20 \mathrm{kHz}$, depending on tape speed. The signal-to-noise ratio of AM recorders tends to be poorer, they have a weaker dynamic response, suffer from greater harmonic distortion and greater wow (distortion due to small irregularities in tape speed).

Another advantage of FM over AM systems is that a tape can be played back at speeds lower than those used for the original recording. In an era when sampling and conversion rates were still slow to reach high scrutinizing speeds ( 2.5 times the maximum frequency to be studied; normally $2,500 \mathrm{~Hz}$ ), this enabled correct transfer of data to a computer. However, playback of tapes at low speeds engenders increased background noise.

\section{Analysis of physiological data}

Analogue processing of signals was normal practice until the late 1970s. The signals were expressed as average acoustic power per frequency band or plotted as wave amplitude against time, sometimes stretching the time axis by recording at higher speeds ("expanded time"). Later improvements in technology made accurate study of the frequency domain of respiratory sounds a more feasible possibility, analysing spectral components of digitized sound samples by the application of Fast Fourier Transform (FFT) algorithms.

Since then, as computers have increased in speed and data handling power, sound recording and electronic processing systems have become entirely digitally based.

Signals are first fed through a signal-conditioner and then through one of two types of fixed frequency sampling devices: 1) a blocking sample device which maintains instantaneous reading values at the A/D converter input; or 2) a multiplexer, consisting of a set of 4-16 input switches, each of which can be routed to a single output and from there to the converter. A decoding subroutine is then required to "demultiplex" the interlaced signals before they can be used.

Prior to digitizing, recorded microphone signals must be played through an antialiasing filter to avoid any unwanted artefacts in frequency spectra. This filter acts as a low-pass filter of analogue signals, and is designed to help counteract beat frequencies that are caused by interference with the sampling frequency of converters. Without such a filter, upper parts of sound spectra would be irreversibly shifted down to lower frequencies and, consequently, cause permanent deformation of signals; sampling will inevitably provoke the phenomenon of aliasing unless frequency bandwidth can be kept within certain limits. According to the Shannon theorem, the ideal sampling frequency $(f \mathrm{~s})$ is 2.54 times the highest signal frequency and, therefore, the best policy would be to discount all data generated by that part of the spectrum lying above $f \mathrm{~s} / 2.54$.

Antialiasing filter cut-off frequencies and sampling frequencies vary greatly from one research team to another because breathing sounds are presumed to be contained within the limits of a relatively discrete frequency band. Examples include filters with a $500 \mathrm{~Hz}$ cut-off [27]; 1 $\mathrm{kHz}$ cut-off $[39,44] ; 1.2 \mathrm{kHz}$ cut-off point and digitized at $8 \mathrm{kHz}$ sampling frequency [29]; a cut-off at $2.5 \mathrm{kHz}$ with a sampling frequency of $5 \mathrm{kHz}$ [28]; sampling at 8 $\mathrm{kHz}$ with no antialiasing filter [13].

Authors rarely give detailed information concerning the electronic data processing systems they have used. Mention is sometimes made of difficulties arising from the limited storage capacities for digitized data [29, 34, $39,49-51]$, but the situation has changed radically over the last 4 yrs due to the development of efficient data handling software equipping widely available computers with clock speeds of over $33 \mathrm{MHz}$, capable of writing directly to large-capacity hard discs of $100 \mathrm{Mb}$ or more.

Most research has been concentrated on the frequency domain of respiratory sounds. Digitized microphone signals are normally broken up into blocks of data 
points and spectral components determined by subjecting the block of data to a FFT. Investigators have used various data blocks, whose numbers are powers of 2 , such as: 128 points [13]; 512 points [28]; 1,024 points [21, 29, 34]; 2,048 points [40, 41]; and 4,096 points [22]. Each FFT treated block of data is then used to provide a discrete plot of amplitude versus frequency, where the quality of spectral resolution depends directly upon the number of FFT points per block and is inversely proportional to the sampling frequency. Successive frequency spectra can be averaged over a fixed period of the signal [29, 37, 39] (such as inspiration or expiration), or displayed sequentially as a pseudo three-dimensional plot (amplitude-frequency-time) called a "waterfall" [12, 34]. Alternatively, frequency spectra may take forms inspired by the work of McKusicK et al. [9], who in 1955 represented acoustic amplitude by light intensity values or a scale of different colour tones on a time versus frequency graph $[38,49]$.

Single compact units, known as spectrum analysers, have been commercially available for several years. They combine recording, antialiasing filtering, A/D conversion, chopping, windowing, FFT functions performed almost instantaneously $[39,52,53]$. It remains true, however, that these specialized devices are almost always designed for work with much higher frequencies than those generated by breathing, and are primarily intended for industrial use. Thus, our laboratory, for example, has tended to acquire individual high quality machines to fulfil single tasks, such as recording or preprocessing signals, these separate elements being co-ordinated by a PC-compatible computer [54]. More recently, our laboratory has converted to all-digital recording and processing of respiratory sounds, the whole system piloted by an IBM RISC 6000 workstation [53, 55].

"The signal-to-noise ratio of lung sounds, particularly vesicular breath sounds, can be sufficiently low that high quality equipment and careful matching of the component parts of a system are necessary" [8].

\section{Normal lung sounds}

The types of sound generated by normal spontaneous breathing, whether tracheobronchial or vesicular sounds, differ according to where they are recorded (generally at the trachea or over the thorax) and also vary with the ventilatory cycle. What follows below is a brief outline of these sounds, their sites of production and their causal mechanisms.

\section{Bronchial sounds}

Tracheobronchial sounds heard during spontaneous ventilation are thought to originate in the upper airways, between the nose cavity and principal bronchi. This type of sound, detected over the trachea and larger airways, is characterized by a hollow or "tubular" quality, which, according to Laënnec, clearly demonstrates that "air is passing through a broader channel than the pulmonary alveoli". The importance of these sounds in the diagnosis of lung consolidation (bronchial breathing murmur) has led to much research being carried out on their frequency content, since the beginning of this century. Martini and Mueller (see [56]) provided evidence in 1923 that the site of bronchial breath sound was in airways 4 $\mathrm{mm}$ or larger in diameter, that it contains higher frequency components than vesicular sounds, and that its presence in abnormal locations signifies a continuous infiltration of lung tissue progressing from the periphery 4-5 cm inwards towards the hilum (where airways of this size are found); this is because consolidated tissues act as more efficient sound conductors than healthy lung tissue. Furthermore, it was later confirmed that bronchial sound could be generated without any active contribution from the larynx.

"Modern" phonopneumography has provided answers to many questions but it has also raised a number of controversial issues over the exact composition of sounds heard at the trachea. McKusick et al. [9] in 1955, recorded bronchial breath sounds with frequencies of 60-600 $\mathrm{Hz}$ during inspiration, and up to $700 \mathrm{~Hz}$ during expiration, signal amplitude remaining relatively constant over these ranges. In 1969, ForGaCs [1] subjectively coined the term "white noise" (energy being evenly distributed over a wide range of frequencies) to describe normal breathing sounds recorded close to the subject's mouth or at the trachea. This is confirmed by the chaotic waveform revealed when the amplitude of these sounds is recorded in extended time mode [57]. In 1981, GAVRIELY et al. [36] calculated mean spectral frequencies (inspiration and expiration phases) showing that the log amplitude response curve remained virtually flat from $75 \mathrm{~Hz}$ (high-pass filter cut-off point) to about $900 \mathrm{~Hz}$, before rapidly falling away at higher frequencies. CHARBonneau et al. [29] in 1983, measured spectra on a linear plot and observed maximum amplitudes between 140-200 Hz, followed by an exponential decline to insignificant levels at about $400 \mathrm{~Hz}$. Such flagrant disparities are partly the result of using different graphical projections to represent data, a linear scale being more likely to over accentuate high amplitude responses and underestimate weaker signals.

Sound spectra are clearly linked to respiratory airflow rate, an increase in the latter engendering a marked upward shift in frequencies.

Turbulent flow characteristics are influenced by conduit dimensions, and tracheal dimensions are a function of body height. Children 9 yrs of age have significantly louder sounds than adults, and higher frequencies at given flow [58].

Studies carried out in our laboratory [53], measured frequency responses in healthy subjects at three steady airflow rates $\left(0.4,0.6\right.$ and $\left.0.9 \mathrm{~L} \cdot \mathrm{s}^{-1}\right)$. The subjects were instructed to base their exchanged gas volume displayed in real time on a triangular waveform cue signal when seeking to attain target airflow rates. This technique produces a square waveform response for the instantaneous airflow, with abrupt direction changes at each inversion of the breathing cycle and, above all, long intermediate periods of almost constant airflow at the desired target 
rate. It was observed that: 1) both in inspiration and expiration, spectra were virtually identical and their waveforms were very similar up to $1 \mathrm{kHz}$ in all subjects; 2) amplitude increased in proportion to airflow rate over the frequency range of 100 to about $800 \mathrm{~Hz}$, the relationship being more marked for inspiration spectra; 3 ) during inspiration as well as expiration phases, maximum frequency values shifted upwards as flow rates increased; 4) tracheobronchial sounds were detected from about $100 \mathrm{~Hz}$, their energy being concentrated between 100 and $1,200 \mathrm{~Hz}$; and 5) from 100 to $600 \mathrm{~Hz}$ the response curve was relatively flat, slowly declining between these two frequencies and then falling more sharply to reach base line levels at 1,200-1,800 Hz, depending on the individual subjects measured.

Tracheal auscultation is now widely accepted as an important diagnostic aid in bronchial challenge tests, detection of breathholding in sleep and nocturnal wheezing (sibilant ronchus). Automated auscultation and analysis represent desirable goals for future experimental work, but before they can be achieved it is vital that standard equipment and measurement protocols, as well as reference values for spectral analysis, become firmly established and accepted by all.

\section{Vesicular lung sounds}

Breath sounds heard close to the chest wall were described by Laënnec as "a distinct murmur corresponding to the flow of air into and out of air cells".

Certain 19th century physicians presumed that the attenuated vesicular sounds heard at the chest wall originated solely in the larynx, whereas other physicians suggested that these sounds were caused by air passing from narrow spaces to larger ones [59]. However, accumulated clinical observations and experimental evidence proved in 1984 that vesicular sound is, at least in part, produced locally, deep beneath the chest wall [8]; for instance, vesicular sounds can be heard over a herniated lung during a Valsalva manoeuvre and also in laryngectomized patients. In vitro experiments showed that vesicular breath sounds occurred even when the trachea was plugged. They also showed that when a sheep's lung was pressed tightly against a human subject's neck, the sounds emitted at the subject's trachea and heard through this artificial barrier bore no resemblance to normal vesicular sounds habitually heard over the thorax. In particular, the transmitted sounds contained no lower frequencies.

McKusick et al. [9] in 1955 were the first to publish representations of vesicular breath sounds as functions of amplitude and frequency. They recorded significant amplitude over a range of frequencies from 0 to $400 \mathrm{~Hz}$ during the inspiratory phase and only a weak response during expiration, composed mostly of sounds from the heart. He concluded that inspiratory vesicular sound resulted from "turbulence created when air currents spread out into the myriads of air sacs..." whilst expiratory sounds had their origins "at bifurcations in the larger airways".
A direct correlation exists between sound intensity and respiratory airflow rate during inspiration, independent of a subject's lung capacity or body position [24, 60-62]. Inspiratory sounds are louder over the lung apex than over the base, given a constant airflow rate.

Frequency spectra measured in healthy subjects show an exponential fall in amplitude starting at $50-75 \mathrm{~Hz}$ and extending to $350 \mathrm{~Hz}$ [39]; $500 \mathrm{~Hz}$ [36] or $1,000 \mathrm{~Hz}$ [35], depending on the results published by different authors. Certain spectra contain numerous peaks or dominant frequencies [35, 39].

The frequency distribution of vesicular lung sound spectra differs between children and adults [63]. Median values of the total frequency range tend to decrease significantly with increasing age, and the exponential fall in intensity over higher frequencies seen in adults can be distinguished from that observed in infants and young children up to the age of 9 yrs [64].

The above results have all been confirmed by experiments on healthy subjects in our own laboratory and, in addition, recording lung sounds along a left-right axillary axis gave rise to the following observations: 1) signal amplitude appeared higher during inspiration, in contrast to normal results obtained in bronchial breathing sounds; 2) absolute amplitude values were lower than those found in bronchial breathing and spectra differed slightly; and 3) vesicular breath sounds were clearly distinguishable at $100 \mathrm{~Hz}$ but amplitude fall-off to baseline values at around 900 or $1,000 \mathrm{~Hz}$ was much more rapid than for bronchial sounds; frequency maxima were lower during expiration.

Similarly, recordings made over the base of the lung show that there is a linear increase of signal amplitude and frequency maxima as respiratory airflow rate rises, the effect being more pronounced during inspiration. Airflow rate may be defined by its two component factors, tidal volume and breathing rate, yet it appears that neither is singly responsible for the above observations and only airflow rate itself can be related to variations in vesicular sound intensity heard at the chest wall.

Amplitude varies greatly from one subject to another ventilating at similar airflow rates and even correction for height, body surface area and weight of subjects is unsuccessful in abolishing this variability.

In an attempt to determine whether the origin of vesicular sound lies more centrally or peripherally, KRAMAN and co-workers [11-13, 45, 65] developed a technique called "subtraction phonopneumography", in which two identical microphones were placed at separate positions along a horizontal line running across the subject's thorax. Concordance of signals from both microphones was taken to mean that the sound source must be situated at a distance, but any time-shift or difference between the two sets of signals was a sign that the source lay within the local area covered by one or both microphones. Using a "subtraction intensity index" (or "cancellation index"), Kraman was able to localize the origin of inspiration-phase sounds as being within the lungs and expiration-phase sounds as coming, at least partly, from the upper airways. 
Similar experimental methods were performed in our laboratory, but they were supplemented by a more powerful data-processing technique in which a "coherence function", measuring the degree of correlation between the two sets of microphone signals, was applied to each individual frequency [53-55]. Good correlation was obtained between $50-600 \mathrm{~Hz}$ with microphones $6 \mathrm{~cm}$ apart, only between $100-150 \mathrm{~Hz}$ with microphones placed $12-18 \mathrm{~cm}$ apart, and no correlation at all with microphones situated on opposite sides, right and left, of the thoracic cage. These results point to a local intralobar or even intralobular site responsible for the major components of vesicular lung sounds and, perhaps, a more centrally situated low-frequency source. The same coherence function applied to lung sounds from tracheal and right posterior basal regions, revealed no correlation between $500-800 \mathrm{~Hz}$ during the inspiration phase and a weak correlation during the expiration phase. It therefore seems unlikely that tracheal sounds contribute to vesicular sounds heard over the base of the lungs. On the other hand, tracheobronchial sounds constitute a significant element of sounds heard over the lung apex.

FORGACS [1] originally attributed the physical cause of these sounds to turbulence in the airstream flowing through the pharynx, larger airways, and as far as the main bronchi. However, his hypothesis soon had to be abandoned. HARDIN and PATTERSON [66] modelled the turbulence that should occur at all bronchial junctions down to the 13th level and calculated their results for the frequency range habitually observed in vesicular lung sounds. Few authors have accepted the proposition that lung tissues may be the physical cause of vesicular sounds. In 1884, Bullar submitted calf lungs to volume and pressure changes in vitro and demonstrated that, in the absence of airflow, no sound would be produced. However, results of our experiments over the last few years have shown that slightly weaker amplitude signals but essentially similar in character to vesicular spectra can be detected over the base of the lung during gentle breathing with the glottis closed $[53,55]$. Such observations may be due to a redistribution of gas within the lungs from apex to base, where the negative pressure exerted by the diaphragm is greater, or maybe the presence of vibrations caused by stretching of lung tissue. Further studies are clearly needed to elucidate the true mechanisms involved in generating vesicular lung sounds.

\section{Normal lung sounds considered globally}

Many physicians use lung sounds, particularly for nursing of patients at hospital, without needing to separate tracheobronchial sounds and vesicular lung sounds. As was seen above, differences in instrumentation, treatment and presentation of acquired data render comparison of individual reports more difficult. The need for agreement over standardization of recording and informationprocessing techniques will be increasingly obvious [67]. Lung sound spectra should be averaged at known airflows over several breaths, and all measurements should be reported relative to sounds recorded at zero flow. But even when the problem of choice in instrumentation and procedures is solved, the question of repeatability of measurements of normal lung sound remains. The stability of lung sounds measurements over time may influence their clinical usefulness [68]. The temporal variability of the spectral pattern of normal lung sounds was assessed in a 30 min interval between recordings of the same session and with a time interval of 1 week. This temporal variability is more significant at the expiratory phase than at the inspiratory phase, and more significant between two recording sessions with a 1 week time interval than between a daily duplicate of the same session. The spectral pattern of normal lung sounds is stable on the trachea and at other locations over the chest wall (right anterior chest - right and left posterior bases of the lungs) except at the interscapular region on the right paravertebral line, where the temporal variability is high [68].

Thus, the future of phonopneumography will be determined by improvements in instrumentation and, furthermore, by increasingly strict standardization of procedures.

\section{References}

1. Forgacs P. Lung sounds. Br J Dis Chest 1969; 63: 112.

2. Forgacs P, Nathoo AR, Richardson HD. Breath sounds. Thorax 1971; 26: 288-295.

3. Cugell DW. Sounds of the lungs. Chest 1978; 73: 311-312.

4. Lyons AS, Petrucelli J. Histoire illustrée de la médecine. Paris, Presses de la Renaissance, 1979.

5. Reiser SJ. The medical influence of the stethoscope. Sci Am 1979; 240: 148-156.

6. Wooten FT, Waring WW, Wegmann MJ, Anderson WF, Conley JD. Method for respiratory sound analysis. Med Instrum 1978; 12: 254-257.

7. Charbonneau G, Sudraud M. Mesure de la réponse en fréquence de quelques stéthoscopes usuels. Conséquences pour l'auscultation cardiaque et pulmonaire. Bull Eur Physiopathol Respir 1985; 21: 49-54.

8. Loudon R, Murphy RLH. Lung sounds. Am Rev Respir Dis 1984; 130: 663-673.

9. McKusick VA, Jenkins JT, Webb GN. The acoustic basis of the chest examination: studies by means of sound spectrography. Am Rev Tuberc 1955; 72: 12-34.

10. Mussel MJ. The need for standards in recording respiratory sounds. Med Biol Eng Comput 1992; 30: 129_ 139.

11. Kraman SS. Determination of the site of production of respiratory sounds by substraction phonopneumography. Am Rev Respir Dis 1980; 122: 303-309.

12. Kraman SS. Does the vesicular lung sound come only from the lungs? Am Rev Respir Dis 1983; 128: 622626.

13. Kraman SS. Lung sounds: relative site of origin and comparative amplitudes in normal subjects. Lung 1983; 161: 57-64.

14. Gavriely N, Palti Y, Alroy G, Grotberg JB. Measurement and theory of wheezing breath sounds. J Appl Physiol: Respirat Environ Exercise Physiol 1984; 57: 481-492.

15. Krumpe PE, Hadley J, Marcum RA. Evaluation of bronchial air leaks by auscultation and phonopneumography. Chest 1984; 85: 777-781. 
16. Olson DE, Hammersley JR. Mechanisms of lung sound generation. Semin Respir Med 1985; 6: 171-179.

17. Waring WW, Beckerman RC, Hopkins RL. Continuous adventitious lung sounds: site and method of production and significance. Semin Respir Med 1985; 6: 201209.

18. Forgacs P. The functional basis of pulmonary sounds. Chest 1978; 73: 399-405.

19. Cohen A, Landsberg D. Analysis and automatic classification of breath sounds. IEEE Trans Biomed Eng 1984; 31: $585-590$.

20. Cugell DW. Lung sounds: classification and controversies. Semin Respir Med 1985; 6: 180-182.

21. Mussel MJ, Nakazono Y, Miyamoto Y, Okabe S, Takishima T. Distinguishing normal and abnormal tracheal breathing sounds by principal component analysis. Jpn J Physiol 1990; 40: 713-721.

22. Lessard C, Jones M. Effects of heart valve sounds on the frequency spectrum of respiratory sounds. Innov Tech Biol Med 1988; 9: 116-123.

23. Druzgalski CK, Donnerberg RL, Campbell RM. Techniques of recording respiratory sounds. J Clin Eng 1980; 5: 321-333.

24. Leblanc P, Macklem PT, Ross WRD. Breath sounds and distribution of pulmonary ventilation. Am Rev Respir Dis 1970; 102: 10-16.

25. Cugell DW. Use of tape-recordings of respiratory sound and breathing pattern for instruction in pulmonary auscultation. Am Rev Respir Dis 1971; 104: 948-950.

26. Akasaka K, Konno K, Ono Y, et al. Acoustical studies on respiratory sounds in asthmatic patients. Tohoku $J$ Exp Med 1975; 117: 323-333.

27. Schreiber JR, Anderson WF, Wegmann MJ, Waring WW. Frequency analysis of breath sounds by phonopneumography. Med Instrum 1981; 15: 331-334.

28. Kanga JF, Kraman SS. Comparison of the lung sound frequency spectra of infants and adults. Pediatr Pulmonol 1986; 2: 292-295.

29. Charbonneau G, Racineux JL, Sudraud M, Tuchais E. An accurate recording system and its use in breath sounds spectral analysis. J Appl Physiol: Respirat Environ Exercise Physiol 1983; 55: 1120-1127.

30. Grassi C, Marinone G, Morandini GC, Pernice A, Puglisi M. Normal and pathological respiratory sounds analyzed by means of a new phonopneumographic apparatus. Respiration 1976; 33: 315-324.

31. Urquhart RB, McGhee J, Macleod JES, Banham SW, Moran F. The diagnosis value of pulmonary sounds: a preliminary study by computer-aided analysis. Comput Biol Med 1981; 11: 129-139.

32. Heemels JP, Ros HH, Sipkema P, De Vries F. Tracheal sound detector. Med Biol Eng Comput 1986; 24: 182-185.

33. Murphy RLH, Del Bono GA, Davidson F. Validation of an automatic crackle (rale) counter. Am Rev Respir Dis 1989; 140: 1017-1020.

34. Mussel MJ, Nakazono Y, Miyamoto Y. Effect of airflow and flow tranducer on tracheal breath sounds. Med Biol Eng Comput 1990; 28: 550-554.

35. Chowdhury SK, Majumder AK. Digital spectrum analysis of respiratory sound. IEEE Trans Biomed Eng 1981; 28: 784-788.

36. Gavriely N, Palti Y, Alroy G. Spectral characteristics of normal breath sounds. J Appl Physiol: Respirat Environ Exercise Physiol 1981; 50: 307-314.

37. Montpetit MJ, Nachman M, Durand LD. Application of numerical methods for feature extraction from phonopneumograms. J Clin Eng 1985; 10: 339-345.
38. Pasterkamp H, Carson D, Daien D, Oh Y. Digital respirosonography: new images of lung sounds. Chest 1989; 96: 1405-1412.

39. Hallgren RC, Huang SM, McMahon SM, Shockey TP. Breath sounds: development of a system for measurement and analysis. J Clin Eng 1982; 7: 135-141.

40. Banham SW, Urquhart RB, Macleod JES, Moran F. Alteration in the low-frequency lung sounds in respiratory disorders associated with crackles. Eur J Respir Dis 1984; 65: 58-63.

41. Baughman RP, Loudon RG. Quantification of wheezing in acute asthma. Chest 1984; 86: 718-722.

42. Chowdhury SK, Majumder AK. Frequency analysis of adventitious lung sounds. J Biomed Eng 1982; 4: 305312.

43. Peirick J, Shepard JW Jr. Automated apnoea detection by computer: analysis of tracheal breath sounds. Med Biol Eng Comput 1983; 21: 632-635.

44. Fenton RT, Pasterkamp H, Tal A, Chernick V. Automated spectral characterization of wheezing in asthmatic children. IEEE Trans Biomed Eng 1985; 32: 50-52.

45. Kraman SS, Austrheim O. Comparison of lung sound and transmitted sound amplitude in normal men. $A m$ Rev Respir Dis 1983; 128: 451-454.

46. Iyer VK, Ramamoorthy PA, Ploy-Song-Sang Y. Quantification of heart sounds interference with lung sounds. J Biomed Eng 1989; 11: 164-165.

47. Iyer VK, Ramamoorthy PA, Ploy-Song-Sang Y. Autoregressive modeling of lung sounds: characterization of source and transmission. IEEE Trans Biomed Eng 1989; 36: $1133-1137$.

48. Ploy-Song-Sang Y, Iyer VK, Ramamoorthy PA. Characteristics of normal lung sounds after adaptive filtering. Am Rev Respir Dis 1989; 139: 951-956.

49. Kaisla T, Sovijarvi A, Piirila P, Rajala HM, Haltsonen $\mathrm{S}$, Rosqvist T. Validated method for automatic detection of lung sound crackles. Med Biol Eng Comput 1991; 29: $517-521$.

50. Charbonneau G, Sudraud M, Racineux JL, Meslier N, Tuchais E. Forced expiration in normal subjects: is the shape of the flow rate curve related to existence of a wheeze? Chest 1987; 92: 825-831.

51. Charbonneau G, Sudraud M, Soufflet G. Une méthode d'évaluation du débit à partir des sons pulmonaires. Bull Eur Physiopathol Respir 1987; 23: 265-270.

52. Marquet P, Dalmay F, Kiniffo F, Menier R. Bruits et vibrations physiologiques: conception et étude comparative de capteurs. Arch Int Physiol Biochem Biophys 1991; 99: A106.

53. Dalmay F. Acquisition et traitement des bruits d'origine ventilatoire chez l'Homme. Thèse de Doctorat de Génie Biologique et Médical, Université de Paris XII, 1992.

54. Menier R, Dalmay F, Marquet P. Dispositif de détection et d'exploration des bruits physiologiques, notamment de bruits ventilatoires et unité de traitement de ces bruits. Brevet d'invention $n^{\circ}$ FR 91 01901, Inst. Nat. Propr. Indust., 18 II (1991).

55. Marquet P. Propriétés acoustiques et vibratoires du système ventilatoire chez l'Homme. Thèse de Doctorat de Physiologie, Université de Paris V, 1992.

56. McKusick VA. In: Cardiovascular Sound in Health and Disease. Baltimore, USA, Williams and Wilkins, 1958.

57. Murphy RLH, Holford SK, Knowler WC. Visual lungsound characterization by time-expanded waveform analysis. N Engl J Med 1977; 296: 968-971.

58. Sanchez I, Pasterkamp H. Tracheal sound spectra depend 
on body height. Am Rev Respir Dis 1993; 148: 10831087.

59. Murphy RLH. Auscultation of the lung: past lessons, future possibilities. Thorax 1981; 36: 99-107.

60. Kraman SS. The relationship between airflow and lung sound amplitude in normal subjects. Chest 1984; 86: 225-229.

61. Kraman SS. Effects of lung volume and airflow on the frequency spectrum of vesicular lung sounds. Respir Physiol 1986; 66: 1-9.

62. Kraman SS, Wang PM. Airflow-generated sound in a hollow canine airway cast. Chest 1990; 97: 461-466.

63. Pasterkamp H, Fenton R, Tal A, Chernick V. Interference of cardiovascular sounds with phonopneumography in children. Am Rev Respir Dis 1985; 131: 61-64.
64. Hidalgo HA, Wegmann MJ, Waring WW. Frequency spectra of normal breath sounds in childhood. Chest 1991; 100: 992-1002.

65. Kraman SS. Does laryngeal noise contribute to the vesicular lung sound? Am Rev Respir Dis 1981; 124: 9294.

66. Hardin JC, Patterson JL. Monitoring the state of human airways by analysis of respiratory sound. Acta Astronautica 1979; 6: 1137-1151.

67. Pasterkamp H, Kraman SS, De Frain PD, Wodicka GR. Measurement of respiratory acoustical signals: comparison of sensors. Chest 1993; 104: 1518-1525.

68. Mahagnah M, Gavriely N. Repeatability of measurements of normal lung sounds. Am J Respir Crit Care Med 1994; 149: 477-481. 